\title{
Flat-Band Ferromagnetism in Organic Polymers Designed by a Computer Simulation
}

\author{
Yuji Suwa, ${ }^{1}$ Ryotaro Arita, ${ }^{2}$ Kazuhiko Kuroki, ${ }^{3}$ and Hideo Aoki ${ }^{2}$ \\ ${ }^{1}$ Advanced Research Laboratory, Hitachi Ltd., Hatoyama, Saitama 350-0395, Japan \\ ${ }^{2}$ Department of Physics, University of Tokyo, Hongo, Tokyo 113-0033, Japan \\ ${ }^{3}$ Department of Applied Physics and Chemistry, University of Electro-Communications, Chofu, Tokyo 182-8585, Japan
}

(Dated: October 30, 2018)

\begin{abstract}
By coupling a first-principles, spin-density functional calculation with an exact diagonalization study of the Hubbard model, we have searched over various functional groups for the best case for the flat-band ferromagnetism proposed by R. Arita et al. [Phys. Rev. Lett. 88, 127202 (2002)] in organic polymers of five-membered rings. The original proposal (poly-aminotriazole) has turned out to be the best case among the materials examined, where the reason why this is so is identified here. We have also found that the ferromagnetism, originally proposed for the half-filled flat band, is stable even when the band filling is varied away from the half-filling. All these make the ferromagnetism proposed here more experimentally inviting.

PACS numbers: 75.10.Lp, 71.20.Rv, 71.10Fd
\end{abstract}

\section{INTRODUCTION}

Materials design to realize desired properties in condensed-matter physics is becoming increasingly realistic. One important factor is, given high levels of computer performance, the computer simulation is an essential part in designing. Namely, computer simulations with first principles calculations should be imperative in narrowing down, or even pin-point, from a wide variety of candidate or designed materials for the interesting properties and functions we search for. Such an approach is especially promising for molecules and nanostructures. While the atom manipulation with scanning tunneling microscopy (STM) ${ }^{1,2.3 .4}$ provides new possibilities for materials design, the design of molecules, especially polymers as we focus on here, should be fundamental.

In this context organic molecules and polymers are of special interest, since they have versatile structures and chemical properties that can be wider than in inorganic materials. Indeed, the discovery of a conducting organic polymer by Shirakawa ${ }^{5.6}$ kicked off intensive studies that are paving a new way to adopt organic polymers or oligomers in realizing various functions as molecular-electronics devices such as field-effect transistors ${ }^{7}$ or light-emitting diodes ${ }^{8}$.

Organic ferromagnets have also attracted much attention as a challenging target ${ }^{9.10}$. In particular, organic magnets consisting entirely of non-magnetic elements is of fundamental as well as practical interests. Ordinary ferromagnets consisting of magnetic elements exploit electrons in d- or f-orbitals that are strongly interacting. Can magnetism arise in p-electron systems that are weakly interacting? One theoretical possibility is to apply the flat-band ferromagnetism proposed by Mielke and by Tasaki11.12. This arises as an effect of electronelectron repulsion when the (one-electron) band structure contains a dispersionless band. The mechanism is interesting in many ways, but essential features are, first, the system is totally distinct from the "narrow-band limit" in textbooks, since the magnetism occurs when the transfer between different sites is finite.

Second, this is a band ferromagnetism rather than a magnetism arising from the exchange interaction between localized spins. So, this mechanism should be a good candidate for designing organic ferromagnets. While there are also other possible mechanisms such as the intramolecular Hund's coupling for spins in p-orbitals $\frac{9}{}$, the flat band ferromagnetism is advantageous in that spins are carried by itinerant electrons which can be utilized for spin injectors in spintronics.

Here a theoretical remark on the flat-band ferromagnetism is due. The flat-band ferromagnetism was first proposed by Lieb, 13 who considers bipartite lattices (consisting of two sublattices) that have different numbers of A sublattice sites $N_{A}$ and B sublattice sites $N_{B}$. Lieb proved that, when we switch on the electron-electron repulsion (assumed to be short-ranged, so that we take the Hubbard model), the ground state should then be ferrimagnetic with the magnetization $\propto N_{A}-N_{B}$. Quantum chemically, this model contains $N_{A}-N_{B}$ nonbonding molecular orbitals, so is similar to Mataga's model. Shima and Aoki14 then proposed a systematic way to realize such systems as superhoneycomb structures. Mielke $e^{15,16,17}$ and independently Tasaki 18 then constructed other flat-band ferromagnetism, which is distinct from Lieb's in that the flat band is constructed from quantum mechanical interference between the nearestneighbor and further transfers (so the system is necessarily non-bipartite) and the ground state is now ferromagnetic.

To be more precise, the lattice is required to satisfy what is called the "local connectivity condition". Namely, the flat band is by no means a sufficient condition for ferromagnetism, and the magnetism arises for special lattices on which adjacent "Wannier" orbitals have to overlap with each other no matter how they are combined to minimize each orbit size. So, while ordinarily a flat band implies a disjointed set of orbits, they are connected in Mielke-Tasaki lattices. This is intuitively 
why spins align to lower the repulsion energy from Pauli's principle. Rigorous proof for the ferromagnetic ground state has been given for the half-filled flat band.

In actual materials, it is very hard to realize perfectly flat bands, and to make them perfectly half-filled. For the former, a rigorous proof (for Mielke-Tasaki lattice) 19 and numerical simulations ${ }^{20.21}$ showed that a slight band dispersion does not destroy the magnetism. For the latter, there are also a proof ${ }^{11}$ and a numerical simulation ${ }^{20}$ which revealed that small deviations from half-filling are allowable for the ferromagnetism.

In the context of this background, what kind of organic materials are promising for realization of the flatband ferromagnetism? The hardest part is to realize the connectivity condition. In general we have to consider complex lattices such as Kagomé, or lattices having distant-neighbor transfers. However, the condition is easier to satisfy on one-dimensional polymers. For example, Mielke-Tasaki model is realized as a chain of triangles ${ }^{20,22,23}$ So we can concentrate on polymers.

Next comes the choice of the monomers that should be polymerized. Even-membered rings such as benzene are disadvantageous in that antiferromagnetic order tends to occur, which should dominate over the ferromagnetism. So we should opt for odd-membered rings, and the above example of the chain of triangles is indeed an example of this. Since triangular molecules are scarce, we can conclude that we should focus on polymers based on fivemembered rings, such as polypyrrole, polythiophen, etc.

For the computer design, the purpose of the present paper, there are two tasks: (i) to search for the materials that realize the flat band a la Mielke-Tasaki. Since the chain of odd-membered rings is by no means a sufficient (nor necessary) condition for the flat band, this is an important part.

(ii) Next, we should do a first-principles (spin density functional) band calculations to confirm (a) the flatness of the band and the ferromagnetism in the ground state, and (b) whether the magnetism can indeed be interpreted as the Mielke-Tasaki mechanism, for which we have to evoke an electron-correlation calculation (exact diagonalization of the Hubbard model here). (c) We also address the question of how the departure from half-filling affects the magnetism in the flat band.

For the first-principles calculation, we have employed the generalized gradient approximation ${ }^{24}$ (GGA). Depending on the purpose of each calculation, we have performed either the spin-unpolarized case based on the density functional theory (GGA-DFT) or the spinpolarized one based on the spin density functional theory (GGA-SDFT). We used the plane-wave-based ultrasoft pseudopotentials ${ }^{25,26}$. The energy cutoff was taken as 20.25 Rydberg. The convergence criterion of the geometry optimization was that all of the forces acting on each atom were within $1 \times 10^{-3}$ Hartree/a.u.

The plan of this paper is as follows. In section III we describe the search for various materials and show some examples which exhibit possibilities of ferromagnetism.
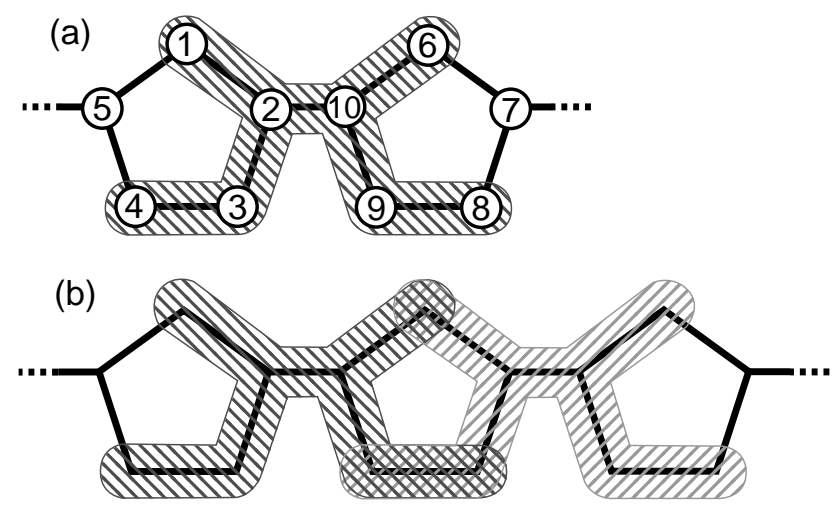

FIG. 1: Schematic view of (a) the eigenfunction that satisfies the connectivity condition and (b) the overlap of the adjacent eigenfunctions.

In section III we show the result for the most promising material, poly-aminotriazole, and discuss how the theory for the flat-band ferromagnetism works on this particular material. In section IV we discuss how the ferromagnetic state is robust against the deviation of the band filling from the half-filling.

\section{EXAMINED MATERIALS}

\section{A. Flat bands in the polymer of five-membered rings}

Let us first examine the simple tight-binding model,

$$
H=-t \sum_{\langle i, j\rangle} c_{i}^{\dagger} c_{j}+\varepsilon_{0} \sum_{i=2}^{5} n_{i}+\varepsilon_{1} n_{1},
$$

for a chain of five-membered rings, where we first assume that all the transfer integrals within the ring and those connecting rings have the same $t$, all the on-site energies are the same $\left(\varepsilon_{0}=0\right)$, except for the one at the top of the ring $\left(\varepsilon_{1}\right)$. We first consider the half-filled case where one electron per site on average. This is a reasonable assumption when we consider $\pi$-orbital networks on such polymers.

Let us start from an observation that Mielke-Tasaki's condition is satisfied when $\varepsilon_{1}=t(>0)$, a not unrealistic condition. In this case, the third band with $E=0$, i.e., the half-filled band, has no dispersion. This provides a heuristic example for the Mielke-Tasaki eigenfunctions: one can construct the most compact eigenfunction which consists of two rings as depicted in Fig. 1(a), where the amplitudes of the numbered sites are given as $(1,1,0$, $-1,0,-1,0,1,0,-1)$. The fact that the amplitudes at the sites 5 and 7 are zero ensures the localized nature of this eigenfunction. Note that we are not displaying a part of a Bloch function, but the whole eigenfunction. One can 


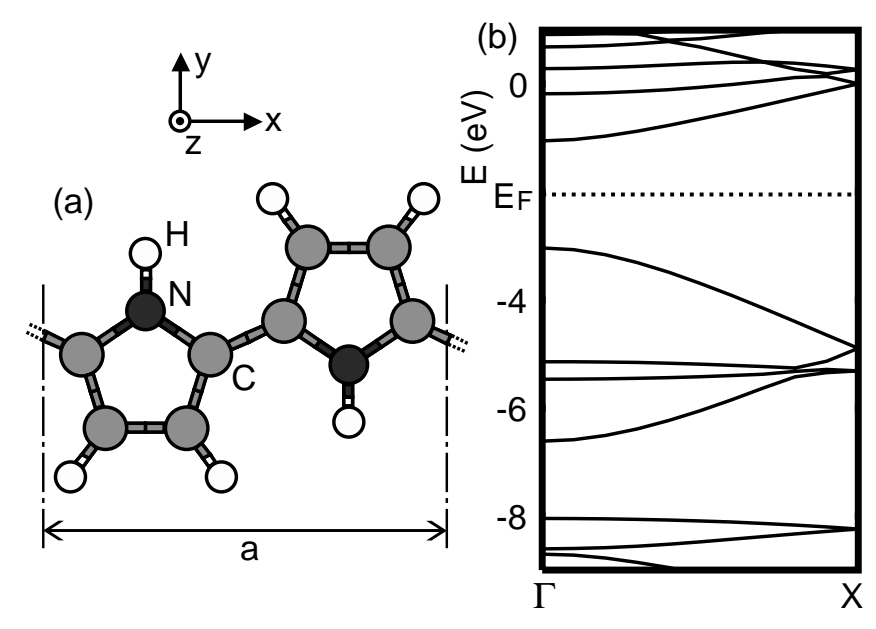

FIG. 2: (a) An atomic structure of polypyrrole. The structure extends along the right and left directions periodically. (b) The (GGA-DFT) band structure of polypyrrole.

also construct another, linearly-independent eigenfunction by simply shifting the wave function from one ring to the next. The eigenvalue of the new function is the same $(E=0)$. So the set of all these eigenfunctions can be a basis for the flat band. Because the eigenfunction is not confined in a single ring (unit cell) but extends over the two rings, two neighboring bases (eigenfunctions) always overlap with each other as shown in Fig. 1(b). One cannot remove such overlaps between the bases no matter how the linear combination of these bases are taken. This overlap is the origin of the ferromagnetic coupling between the electrons in the flat band.

\section{B. Known polymers of five-membered rings}

A typical polymer of five-membered rings is polypyrrole. The atomic structure and calculated band structures are shown in Figs. 2(a) and (b), respectively. The five-membered rings alternate their directions in this compound, so that the unit cell contains two rings. $\mathrm{X}$ point in Fig. 2(b) has $\boldsymbol{k}=(\pi / a, 0,0)$, where $a$ is the lattice constant of this unit cell as shown in Fig. 2(a). Because of the doubled unit cell, most of the bands are folded at $\mathrm{X}$. For directions $(y, z)$ perpendicular to the chain, we have taken a cell size large enough to avoid inter-chain interactions. We can see that polypyrrole has, not surprisingly, no flat bands around the Fermi energy.

We have also looked at other typical polymers of fivemembered rings, including polythiophen (where an $\mathrm{N}-\mathrm{H}$ block in polypyrrole is replaced with an $\mathrm{S}$ atom) and polytriazole (where all the $\mathrm{C}-\mathrm{H}$ blocks in polypyrrole are replaced with $\mathrm{N}$ atoms). However, their band structures have no flat bands, either.

\section{Designing the polymer}

This is exactly where the designing comes in. Our strategy consists of two approaches. One is to replace $\mathrm{H}$ atom bound to $\mathrm{N}$ atom at the top of the ring with various kinds of bases. According to the tight-binding calculation described in the previous subsection, the onsite energy $\left(\varepsilon_{1}\right)$ of the top of the ring should be higher than that of $\mathrm{C}$ atoms to have a flat band. Because the on-site energy of $\mathrm{N}$ is expected to be lower than that of $\mathrm{C}$, the first attempt should be the replacement of the $\mathrm{N}$ atom with other elements with higher on-site energies. However, that should be difficult if one wants to retain the existence of the $\pi$-orbital and single electron occupation on it. Therefore, we have opted for controlling the on-site energy by replacing the $\mathrm{H}$ atom with other functional groups, by leaving the $\mathrm{N}$ atom intact.

Alternatively, we can replace $\mathrm{C}-\mathrm{H}$ blocks on the bottom edge of the pentagons (3, 4, 8, 9 in Fig. 1(a)) with $\mathrm{N}$ atoms, where the lowering of the on-site energies of the bottom of the ring may effectively realize the required condition. In other words, we use polypyrrole and polytriazole as the platform to modify either the top or the bottom of the ring. Since it is not obvious which approach should be better, we consider both platforms for all the substituents considered here.

Substituents we have tested are sodium (Na), potassium $(\mathrm{K})$, chlorine $(\mathrm{Cl})$, fluorine $(\mathrm{F})$, cyanogen $(\mathrm{CN})$, nitro $\left(\mathrm{NO}_{2}\right)$, sulfate $\left(\mathrm{SO}_{4}\right)$, carboxyl $(\mathrm{COOH})$, amino $\left(\mathrm{NH}_{2}\right)$, methyl $\left(\mathrm{CH}_{3}\right)$, and hydroxyl $(\mathrm{OH})$. For all these candidates, we have performed first-principles (GGADFT) optimization of atomic structures, and obtained their band structures. Most of them have no flat bands around $E_{F}$, but some of them have turned out to have flat bands. For these we have performed further (GGASDFT) first-principles optimizations under the doping condition to make the flat band half-filled.

First, we have examined single atoms (alkali metal or halogen) as substituents. This is motivated by the fact that small substituents may be better than larger ones, because a bulky group at the top of the ring may introduce a coupling (transfer) with the bottom of the adjacent ring, which would destroy the basic assumption that the adjacent rings are connected by one bond to satisfy Mielke-Tasaki's condition.

In order to raise the on-site energy of the top of the ring, we have first considered an alkali-metal atom, $\mathrm{Na}$, because of its low electron affinity. Figure 3(a) shows the atomic structure of poly-sodium-triazole, where hydrogen atoms in polytriazole are replaced by sodium atoms. Figure 3(b) shows the band structure, where one can see that a nearly flat band exists above the Fermi energy. Absence of any unnecessary bands between the flat band and $E_{F}$ is good sign, because we can dope carriers into the flat band without any complications.

We have also examined the Na-substituted polypyrrole, and obtained a similar band structure. However, in this case the flat band above $E_{F}$ is much closer to a dis- 

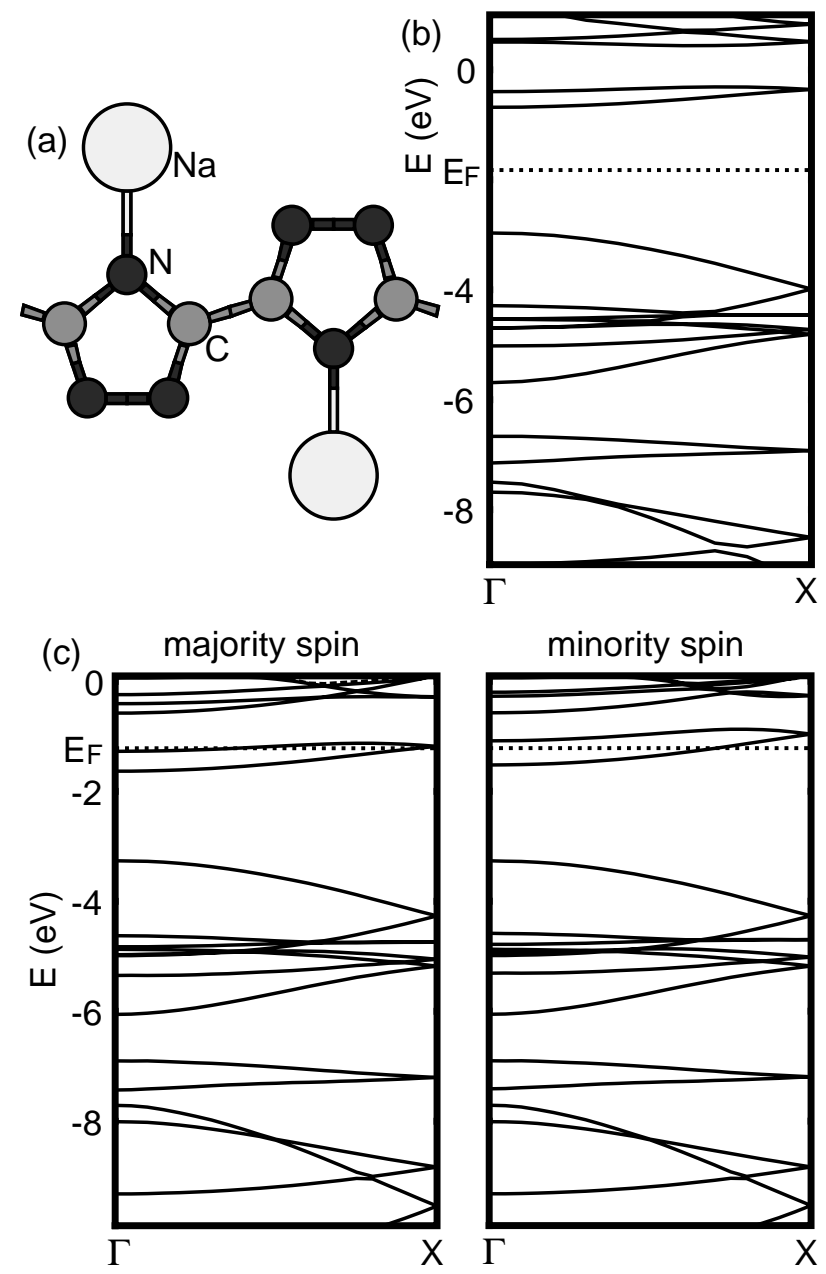

FIG. 3: (a) The optimized atomic structure of poly-sodiumtriazole, the band structure of poly-sodium-triazole when undoped (spin unpolarized)(b), and doped (spin polarized)(c).

persive band below $E_{F}$, which can introduce unwanted inter-band interactions. As for the alkali-metal we have also considered potassium, but the case of $\mathrm{Na}$ has turned out to be better.

We move on to the doping. First, the flat bands always appear as a pair of bands folded at X point, except for the case of antiferromagnetic ordering. Therefore, we make the flat-band half-filled by doping two carriers per unit cell into the pair of flat-bands. Namely, one carrier is doped for each ring. In poly-sodium-triazole, we have considered the doping of two electrons per unit-cell. Here we realize the doping condition by increasing the number of electrons with a uniform positive background charge for charge neutrality.

After a geometrical optimization of doped polysodium-triazole, we have calculated the spin-dependent (GGA-SDFT) band structure in Fig. 3(c). One can see that the number of occupied states differs between majorand minor-spins, which means that the system is ferromagnetic. It should be noted that (the pair of) the flat band is not entirely spin-split. The difference of the number of spins is 0.65 per unit cell. The spin polarization is smaller than the expected polarization (2.0). We also found that the total energy (per unit cell throughout this paper) of the ferromagnetic (F) state is lower by $12 \mathrm{meV}$ than that of the paramagnetic (P) state. We could not find antiferromagnetic (AF) solution even when we started geometry optimization from an AF electronic state. So the F state is the most stable in this material, but is only slightly lower in energy than the $\mathrm{P}$ state.

The reason why this material gives such an insufficient result in spite of the existence of the nearly flat band should be sought in the nature of the flat-band's wave function. We found that the half-filled flat band here is made of an orbital localized around the $\mathrm{Na}$ atom, so does not satisfy the local connectivity condition. This should be why the stability of the F state is very weak, while the finite spin-polarization in GGA-SDFT should be a narrow-band effect rather than a much more robust Mielke-Tasaki effect. Indeed, the difference between the weak ferromagnetism here and a robust ferromagnetism in poly-aminotriazole, shown later, is a good demonstration of the importance of the local connectivity condition, hallmark of the flat band ferromagnetism.

Now we digress a bit, and consider the contrary case of an atom having high electron affinity such as a halogen, e.g., fluorine atom, which might be heuristic. Figure 4(a) shows the atomic structure of poly-fluoropyrrole, where the fluorine atoms are substituted for hydrogen atoms bound to nitrogen atoms in polypyrrole. Figure 4(b) is the band structure obtained by spin-unpolarized (GGADFT) calculation. We find a flat band which lies just in between two dispersive bands below $E_{F}$. Because of this the doping greater than one carrier per unit cell is required.

So we consider making the flat-band half-filled by doping four holes per unit-cell. Here, two holes are necessary to make the dispersive band just below the Fermi level empty, and two holes are necessary to make the folded flat bands half-filled. Figure 4(c) shows spin-dependent band structure after the geometry optimization under the doping condition. The system is seen to be ferromagnetic. The difference of the number of spins per unit cell is just 2.0. One problem of this material, however, is that doping twice as large is necessary. When the doping is performed in this material, the flat band shifts upward (downward) for minority (majority) spin. Replacing the fluorine atom with a chlorine atom results in a band structure similar to the case of fluorine. In fact an AF state, obtained by starting geometry optimization from an antiferromagnetic state, has a total energy 21 meV lower than that of $\mathrm{F}$ state.

We move on to functional groups as substituents to search further possibilities. We have considered an example, poly-hydroxytriazole, where $\mathrm{H}$ atom in polytriazole is replaced by $\mathrm{OH}$ with a low electron affinity. Although a pair of slightly dispersive bands exist just below $E_{F}$, these bands, when doped with two holes per unit cell, 

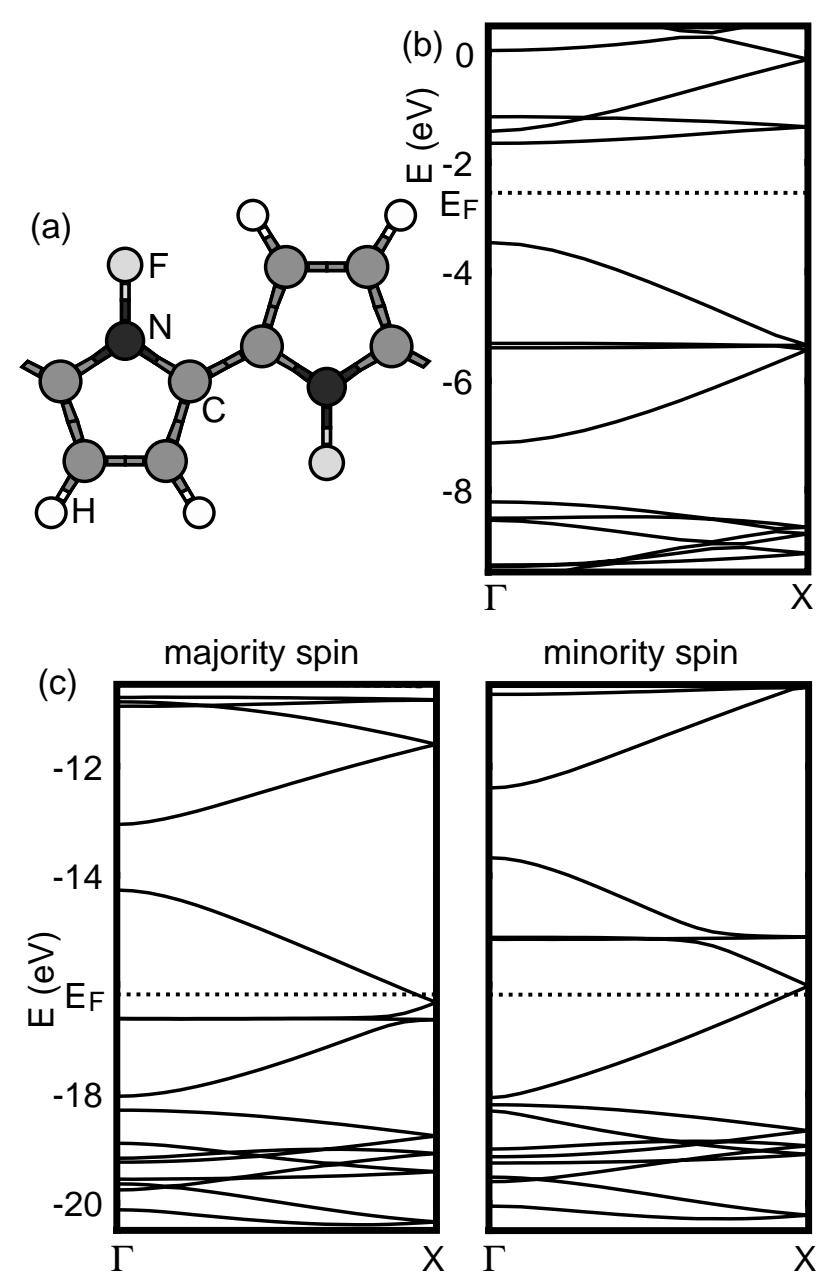

FIG. 4: (a) The optimized atomic structure of polyfluoropyrrole, the band structure of poly-fluoropyrrole when undoped (spin unpolarized)(b), and doped (spin polarized)(c).

become mixed with dispersive bands underneath. As a result, the difference of the number of spins in the $\mathrm{F}$ state is only 0.39 per unit cell, and the paramagnetic state is more stable than the $\mathrm{F}$ state by $3 \mathrm{meV}$.

Figure 5(a) is the atomic structure of polyaminopyrrole, where $\mathrm{H}$ atom bound to $\mathrm{N}$ atom in polypyrrole is replaced by $\mathrm{NH}_{2}$, whose electron affinity is low. In the band structure, Fig. 5(b), one can find a pair of flat bands below $E_{F}$. The lower half of it is very flat while upper half is slightly dispersive.

We have then doped two holes per unit cell. The band structure after geometry optimization is shown in Fig. [5 (c). The difference of the number of spins is 1.0 per unit cell. As one can see from Fig. [5)(c), the Fermi level intersects the middle of the pair of flat bands. Although the lower part of the pair of flat bands is quite flat, the dispersion of the upper half part is too large. Here only lower half part seems to work as a flat-band. The total energy of paramagnetic state in this material is found to
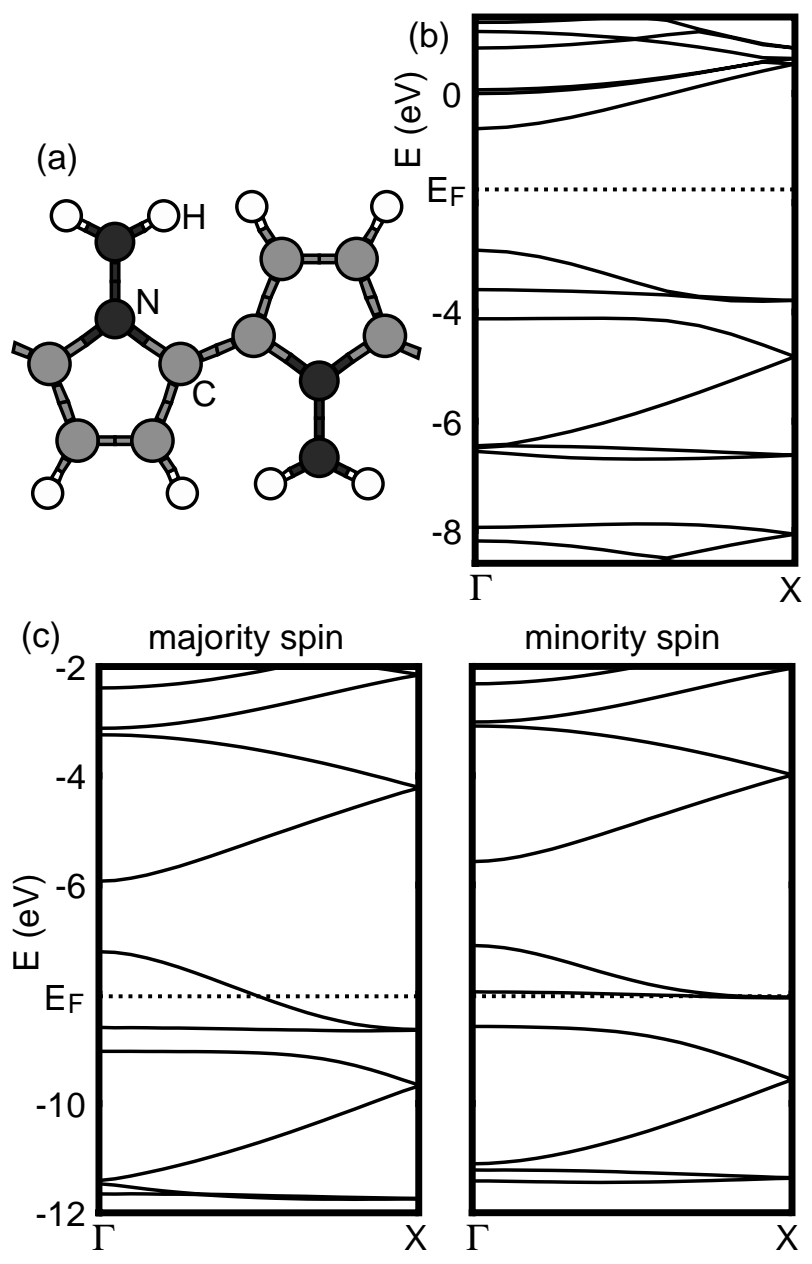

minority spin

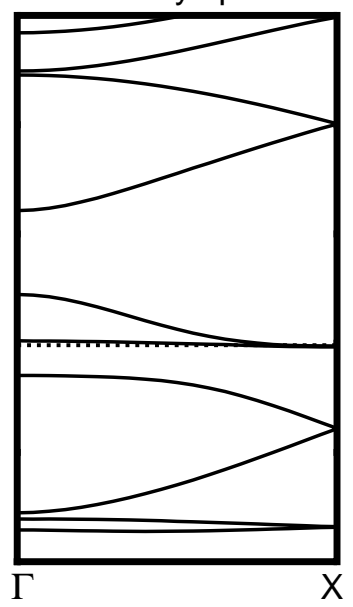

FIG. 5: (a) The optimized atomic structure of polyaminopyrrole, the band structure of poly-aminopyrrole when undoped (spin unpolarized)(b), or doped (spin polarized)(c).

be $65 \mathrm{meV}$ higher than that of ferromagnetic state, while an AF solution does not exist, so the F state is the most stable.

All other substituents tested here except for the one in the next section have turned out to be inappropriate. In most of those materials, the band which we expect to be flat is either too dispersive or too deep. For the remaining ones, band structures are qualitatively similar to the examples described in this section. Poly-aminotriazole, discussed in the next section, also gives qualitatively similar results to poly-aminopyrrole shown in this section, but quantitatively much better.

\section{POLY-AMINOTRIAZOLE}

\section{A. First principles calculation}

The best candidate for ferromagnetic organic polymer among the materials we have considered is poly- 


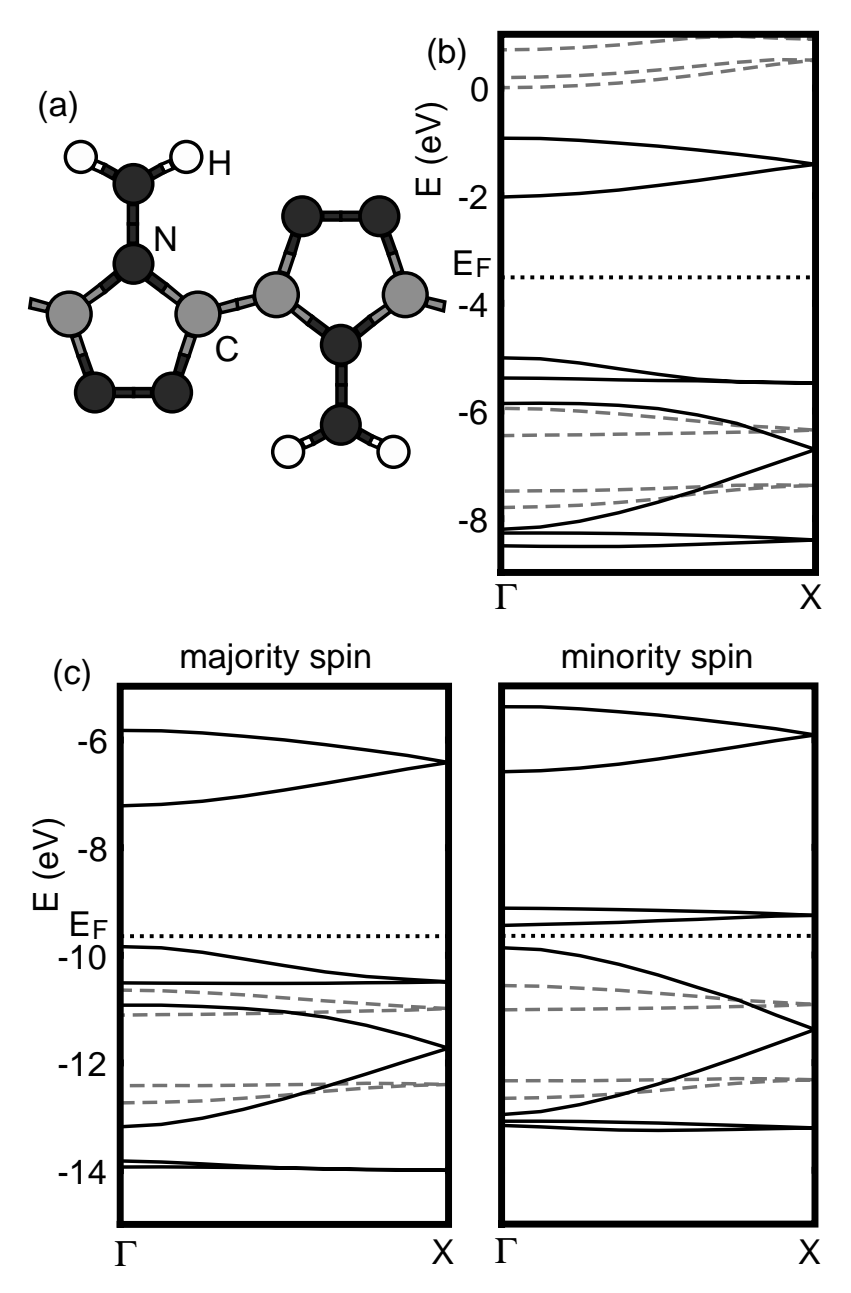

FIG. 6: (a) The optimized atomic structure of polyaminotriazole, the band structure when undoped (spin unpolarized)(b), and doped (spin polarized)(c). The bands having $\pi(\sigma)$ character are shown by solid (dashed) lines, respectively.

aminotriazole 27 . Figure 6(a) shows the atomic structure of poly-aminotriazole, where $\mathrm{H}$ atom in polytriazole is replaced with $\mathrm{NH}_{2}$. The difference between polyaminopyrrole shown in the last section and this material is whether the platform is polypyrrole or polytriazole, i.e., the $\mathrm{C}-\mathrm{H}$ blocks in the bottom of the ring is replaced with $\mathrm{N}$ atoms. The band structure of the poly-aminotriazole, shown in Fig. 6(b), is similar to that of Fig. [5), but the dispersion of the upper half of the pair of flat bands is smaller and the separation from dispersive bands below is greater than that of Fig. 5 (b). These features are desirable for the flat-band ferromagnetism.

After the optimization of the atomic structure under the doping condition of two holes per unit cell, the spin dependent band structure was calculated and shown in Fig. 6(c). The result hits on the ideal situation - The pair of flat bands is made half-filled, and as a result, fully occupied by majority spins while totally unoccupied by minority spins. Reflecting this situation the difference of

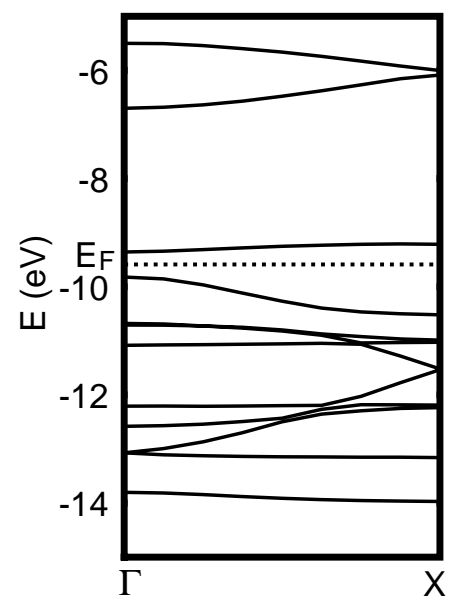

FIG. 7: A band structure for a metastable antiferromagnetic state in poly-aminotriazole.

the number of spins is the desired 2.0 per unit cell.

In this material, we found a metastable antiferromagnetic state. The band structure of the AF state is shown in Fig. (7. This is calculated with GGA-SDFT, but the resulting band structures for up- and down-spins are the same, while the wave functions for up- and down-spins are arranged alternately on the chain of rings. This means that even though we have avoided intra-ring $\mathrm{AF}$ instability by choosing five membered rings, inter-ring $\mathrm{AF}$ can exist at least as a metastable state. The upper and lower parts of the flat band folded at $\mathrm{X}$ point is separated with $E_{F}$ in between. The AF state is found to be $52 \mathrm{meV}$ higher in energy than the F state. Paramagnetic state is even higher (by $384 \mathrm{meV}$ ) than the $\mathrm{F}$ state. Therefore $\mathrm{F}$ state is the most stable in this material.

Let us discuss how the flat-band ferromagnetism is achieved in this material by combining the wave functions of the flat band obtained by first principles calculation with the calculation in the tight-binding model. Figure 8 shows the majority-spin's wave functions in the pair of flat bands just below the Fermi level in Fig. 6(c). While the phases of the wave functions can be taken arbitrarily, we choose for clarity the wave functions of the lower and upper bands at $\Gamma$ point (Figs. 8(a) and (f)) to be real and pure imaginary, respectively, and those at intermediate $k$-points to be continuous. If we first look at the real part, typically for Fig. 8 (a), the wave function for each monomer (aminotriazole) has two nodal lines which divide the amplitude into three parts, corresponding to $\mathrm{NH}_{2}, \mathrm{C}-\mathrm{N}-\mathrm{C}$, and N-N blocks. For the imaginary part, typically for Fig. 8 (f), the wave function has one nodal line, which divides the amplitude into two, consisting of two C-N blocks. The two wave functions (real and imaginary), which are orthogonal, are mixed at the intermediate $k$-points. 

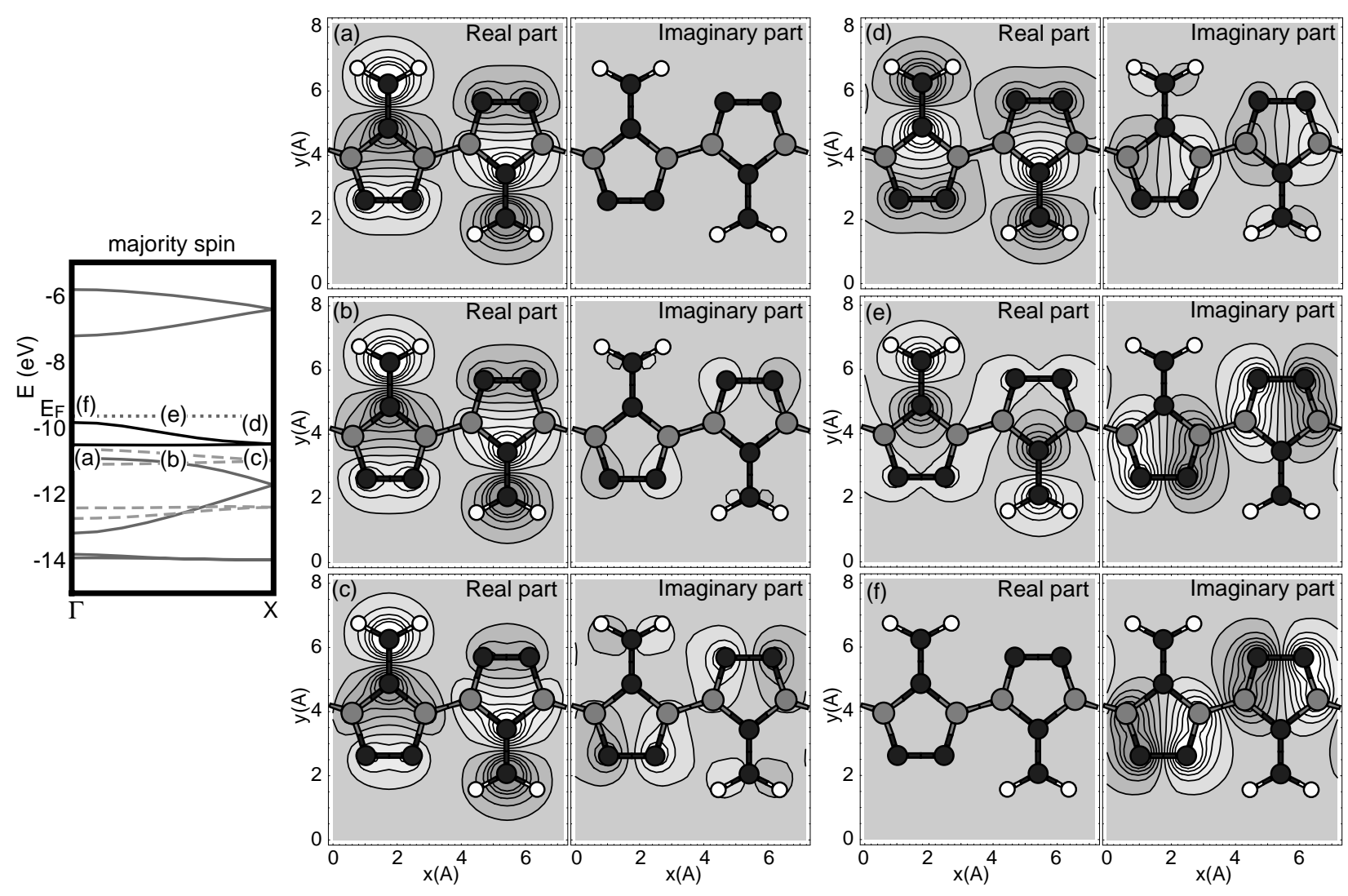

FIG. 8: Wave functions of the polarized lower band $(\mathrm{a}, \mathrm{b}, \mathrm{c})$ and upper band $(\mathrm{d}, \mathrm{e}, \mathrm{f})$ of poly-aminotriazole. (a, f) for $k_{x}=0$ $(\Gamma)$, (b, e) for $k_{x}=\pi / 2 a$, and (c, d) for $k_{x}=\pi / a(\mathrm{X})$. Inset at the left side indicates the band and $k$-point of each figure.

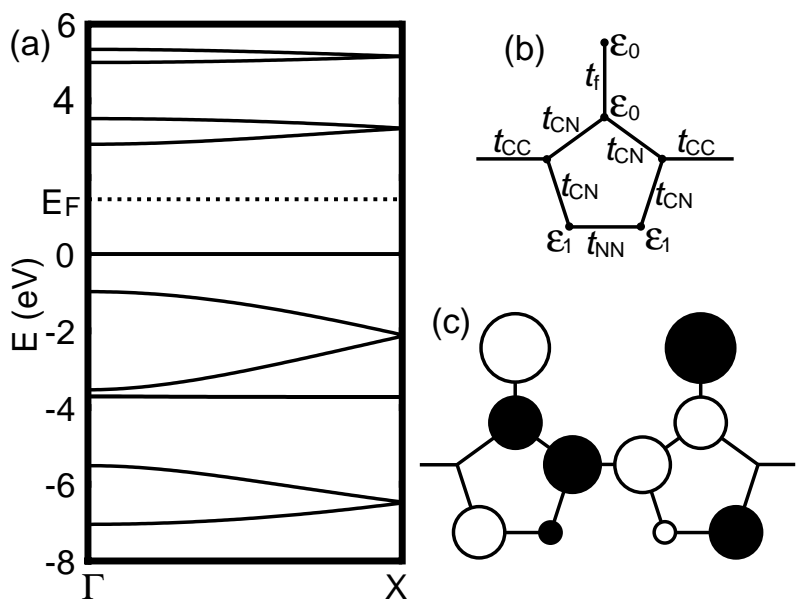

FIG. 9: (a) Band structure in the tight-binding model. (b) Tight-binding parameters used for the fitting. (c) The eigenfunction which satisfies the connectivity condition.

\section{B. Tight-binding model}

In order to capture the essence of the flat band, here we introduce a tight-binding model which represents $\pi$ orbital's network in this system. Figure 9] a) shows its band structure. The parameters of the model displayed in Fig 9(b) are fitted so as to reproduce Fig. 6(b). Obtained values are: $t_{\mathrm{CN}}=t_{\mathrm{CC}}=t_{\mathrm{f}}=2.5 \mathrm{eV}, t_{\mathrm{NN}}=3$ $\mathrm{eV}, \varepsilon_{0}=-1.4 \mathrm{eV}$, and $\varepsilon_{1}=-0.5 \mathrm{eV}$. One can see that the tight-binding band (Fig. 9/a)) excellently agrees with the $\pi$-bands obtained by first-principles calculation (solid lines in Fig. 6(b)).

Figure 91(c) shows the tight-binding wave function of the eigenstate corresponding to that in Fig. 1(a). In this material, one site is added to the top of the ring which represents a nitrogen atom of $\mathrm{NH}_{2}$. The open and closed circles indicate the sign of the wave function, respectively, and their sizes amplitudes. This wave function satisfies the local connectivity condition.

Here it should be noted that the substitution of $\mathrm{NH}_{2}$ for $\mathrm{H}$ not only raises the on-site energy of the top of the ring but also introduces an extra $\pi$-orbital of the $\mathrm{N}$ in $\mathrm{NH}_{2}$. The existence of the extra site completely changes the connectivity condition, so that we have no longer to satisfy $\varepsilon_{1}=t>0$, which has turned out in the present 


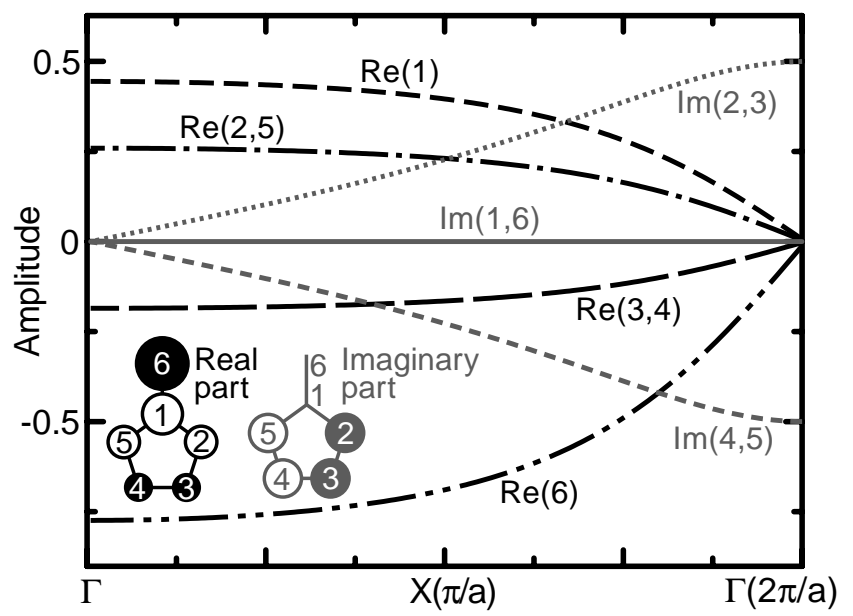

FIG. 10: $k$-dependence of the wave functions in the tightbinding model.

study to be rather difficult condition to realize.

Figure [10 shows $k$-dependence of the tight-binding wave functions of the flat band. Here $k$-space is extended to the second Brillouin zone, so the left and right halves of the figure represent the wave functions of the lower and upper parts of the pair of flat bands, respectively. Phase factors are chosen again for clarity as in Fig. 8 . The label " $\operatorname{Re}(2,5)$ " indicates that the line represents the real part of the wave function at the site 2 and 5 , where the numbering of the sites is shown in the insets. On the $\Gamma$ points at the left- and right-edge of the figure, the wave function becomes real and pure imaginary, respectively. These two wave functions are displayed in the inset of Fig. [10] where it should be noted that we display a part of the periodically extending Bloch functions, in contrast to Fig. 9(c) which shows the entire eigenfunction.

One can see that the symmetry and the character of the wave functions shown in Fig. 10 is similar to the first-principles wave function (Fig. 8). These wave functions can be constructed by a linear combination of the eigenfunctions shown in Fig. 9(c). Figure [1] illustrates how the linear combination of the localized eigenfunctions provides the Bloch function. When the two neighboring eigenfunctions, $\mathrm{A}$ and $\mathrm{B}$, are added, the sum at the ring on which they overlap (enclosed by dotted lines) forms the shape of the imaginary-part wave function as shown in the inset of Fig 10 When subtracted, it forms the shape of the real-part wave function. The entire imaginary- and real-part wave functions are constructed by the summation of all localized eigenfunctions $(\mathrm{A}+\mathrm{B}+\mathrm{C}+\mathrm{D}+\cdots)$ and the combination of the summation and subtraction $(\mathrm{A}-\mathrm{B}+\mathrm{C}-\mathrm{D}+\cdots)$, respectively. The real-part wave function and the imaginarypart wave function are orthogonal to each other. The gradual mixing of these two orthogonal wave functions as $k$ is changed is the very character of the Mielke-Tasaki flat band, which should never occur on an ordinary flat

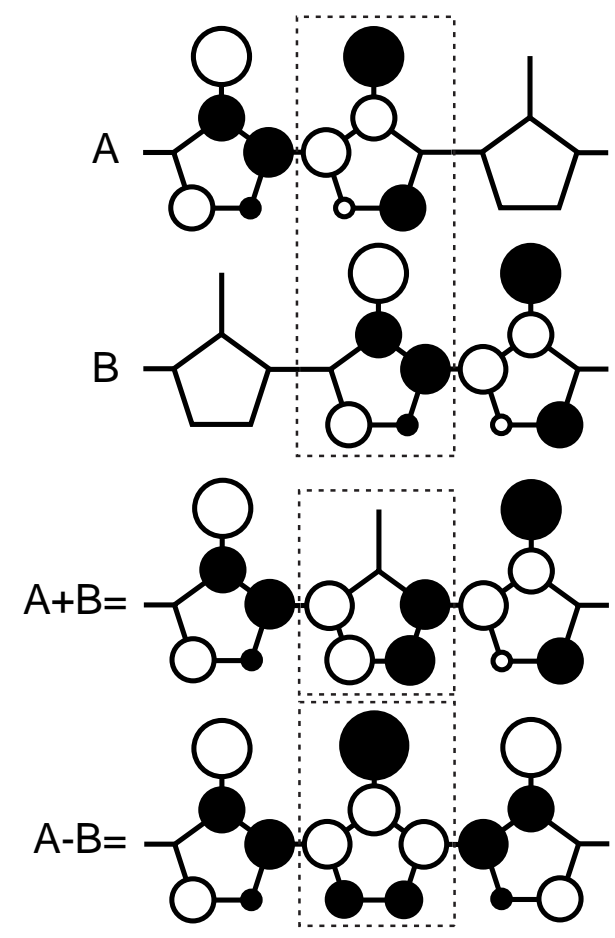

FIG. 11: Schematical linear combinations of the neighboring eigenfunctions.

band.

\section{Comparison with the Hubbard model}

Having constructed the tight-binding modeling we proceed to the question of whether the ground state is spinpolarized in the presence of the Hubbard interaction, $\mathcal{H}_{U}=U \sum_{i} n_{i \uparrow} n_{i \downarrow}$. In Fig. 12 we show the phase diagram against $U$ and $\varepsilon_{1}$ obtained with an exact diagonalization calculation for a 12-site (2 rings) Hubbard model for $t_{0}=2.5 \mathrm{eV}$ and various values of $t_{\mathrm{NN}}=3.0-4.0$ $\mathrm{eV}$. As indicated in the inset, $\varepsilon_{0}$ is chosen throughout to satisfy the condition for the flat band,

$$
\begin{aligned}
\varepsilon-\varepsilon_{1} & =(1-\varepsilon)\left(t_{\mathrm{NN}}^{2}-\left(\varepsilon-\varepsilon_{1}\right)^{2}\right)-t_{\mathrm{NN}}, \\
\left(\varepsilon-\varepsilon_{1}+t_{\mathrm{NN}}\right) /(1-\varepsilon) & =-t_{\mathrm{NN}} /\left(\varepsilon_{0}-\varepsilon\right)-t_{\mathrm{NN}}\left(\varepsilon-\varepsilon_{0}\right),
\end{aligned}
$$

where $\varepsilon$ is the eigenenergy of the flat band and $t_{\mathrm{CN}}=$ $t_{\mathrm{CC}}=t_{0}(=1$ here $)$ is assumed for simplicity. We can see that we have indeed a ferromagnetic phase unless the repulsion is too strong (i.e., $U<U_{c}$ with $U_{c}=2 \sim 5 \mathrm{eV}$ ). Our preliminary quantum Monte Carlo calculation confirms that two unit cell is sufficient to roughly determine the ferromagnetic phase boundary. Peculiar destruction of the ferromagnetism above $U>U_{c}$ is another hallmark of the Mielke-Tasaki magnetism ${ }^{21}$ 


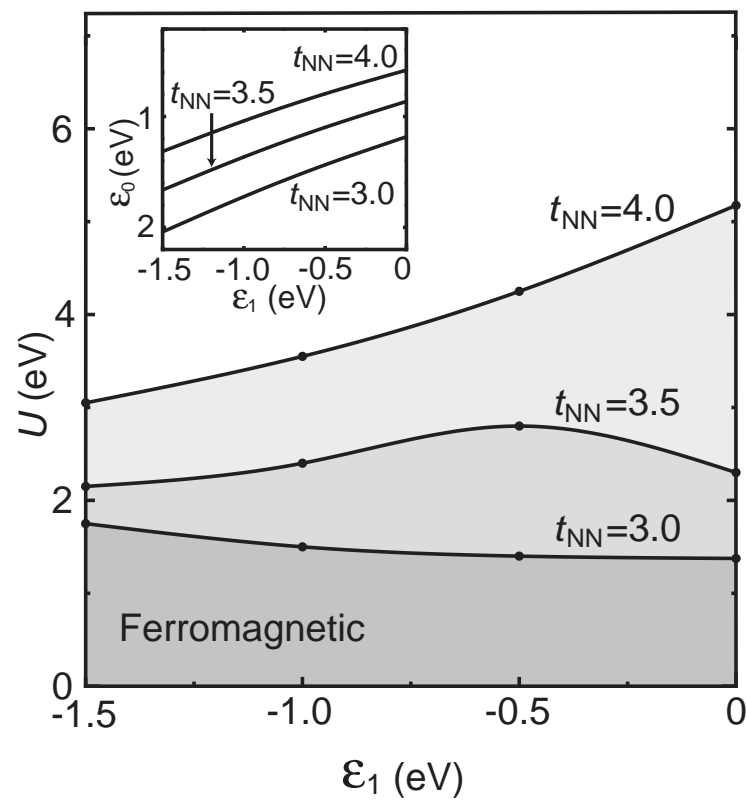

FIG. 12: Stability of the ferromagnetic phase calculated with an exact diagonalization of the Hubbard model.

\section{DEVIATION FROM THE HALF-FILLED FLAT BAND}

\section{A. Comparison of the total energies}

In order to realize ferromagnetism in the materials we proposed here, carriers have to be doped into the flat band. We can consider several possible methods to achieve this such as field effect doping ${ }^{28}$, photo-induced carrier injection $^{29}$, as well as chemical doping. The first two are now attracting much attention in molecular electronics, but they are very recent techniques and may need further developments. We can instead concentrate on the chemical doping, where the polymers are crystallized along with the accepters, and we found it feasible. Details are presented elsewhere ${ }^{30}$. Now, if the flat-band ferromagnetism can occur not only just at the half-filling but also away from that, this would make the chemical doping much easier.

We have to know how robustly the ferromagnetism survives when the filling of the flat band deviates from just half-filled. Although there is a proof ${ }^{11}$ and a numerical study ${ }^{20}$ for a Mielke-Tasaki lattice which show that slight deviations do not wash out the ferromagnetism, those conclusions may not be simply applied to other models. For the present model, calculations taking full account of the electronic correlation such as the exact diagonalization of the Hubbard model would be unsuitable, because changing the number of electrons in small systems corresponds to too big a change in the filling. We have instead performed an SDFT calculation, because it can handle the fractional number of electrons (per unit cell) if a uniform background charge is introduced.

At this point we can note the following. In SDFT calculations, electronic correlations are not fully taken into account, so we can question whether the flat band ferromagnetism may be described within the SDFT framework. This problem has been addressed in our previous publication ${ }^{27}$ where SDFT and exact diagonalization of a Hubbard model have given qualitatively consistent results. This is not surprising, since one of the remarkable features of the flat-band ferromagnetism is that the magnetism exists over the whole range (infinitesimal $<U<\infty)$ of the interaction as proved rigorously, so the weak- $U$ case (for which the methods such as SDFT is meant to be applicable) crosses continuously over to strong- $U$ case. As far as the on-site repulsion is not too strong, the comparison between the gain of the exchangecorrelation energy by spin polarization ( $\mathrm{F}$ state) and the gain of the band energy by forming $\mathrm{AF}$ order (or equivalently, a spin density wave (SDW) with the periodicity of two five-membered rings) accompanied by a lattice relaxation should predominantly determine which state is stable. These two energies can be accurately estimated by SDFT calculations. Therefore, we expect here that SDFT can serve our purpose, to see the band-filling dependence of the total energies of $\mathrm{F}, \mathrm{AF}$, and $\mathrm{P}$ states.

Figure 13] (a) shows band-filling dependence of the total energies of the $\mathrm{F}$ and $\mathrm{AF}$ states of the polyaminotriazole measured from that of the $\mathrm{P}$ state. The position where the number of holes, $n_{h}$, is 2.0 corresponds to the half-filling. The results for F, AF, and $\mathrm{P}$ are obtained by fully relaxing the atomic geometry for each electronic state. It can be seen that $\mathrm{F}$ state is stable in the range $1.1<n_{h} \leq 2.0$, while $\mathrm{P}$ state is stable for $n_{h}<1$.1. In the region where $\mathrm{F}$ is stable, $\mathrm{AF}$ is found to be metastable. In the P-region, $n_{h}<1.1$, no AF state is found. This result shows that the ferromagnetism in this material is robust, even down to the quarter filling, against the deviation from the half-filling. The result that quarter-filling is acceptable greatly relaxes the difficulty in doping.

\section{B. Correlation with the atomic configuration}

Since the polymer proposed here has functional groups, we should also examine how the three-dimensional atomic configuration is correlated with the band-filling dependence. Namely, all the atoms do not lie on a plane: in poly-aminotriazole two hydrogen atoms in $\mathrm{NH}_{2}$ in the undoped polymer lie above the plane of the rings, while all the atoms are co-planar in the half-filled polyaminotriazole. We can more closely look at the torsion angle, $\phi$, defined as the angle with which $\mathrm{C}-\mathrm{N}-\mathrm{N}-\mathrm{H}$ atoms are connected. Figure 13(b) shows the torsion angles in the $\mathrm{P}$ and $\mathrm{F}$ states, respectively. From Figs. 131(a) and (b), one can see that the breakdown of the ferromagnetism with the decrease in the number of holes is correlated with the change in the atomic configuration. 


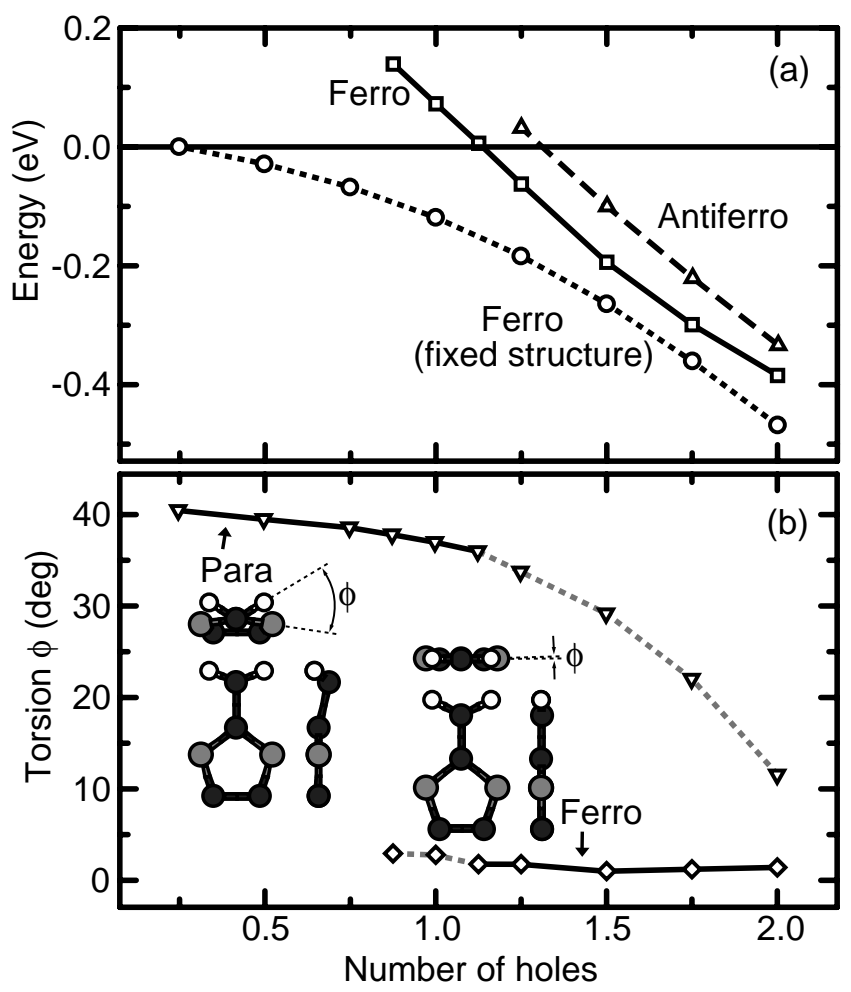

FIG. 13: Doping dependence of (a) the total energy (relative to the paramagnetic state) of $\mathrm{F}$ (solid line), $\mathrm{AF}$ (dashed line), and $\mathrm{F}$ (fixed structure, dotted line, see text) states measured from that of $\mathrm{P}$ state, and (b) torsion angle of $\mathrm{C}-\mathrm{N}-\mathrm{N}-\mathrm{H}$ in $\mathrm{F}$ (diamonds) and $\mathrm{P}$ (reversed triangles) states. Stable states are connected by a solid line, while metastable states by a dotted line. Insets show front, side, and top views of the atomic structure of the aminotriazole in $\mathrm{P}$ and $\mathrm{F}$ states.

When the system is half-filled $\left(n_{h}=2.0\right)$, all atoms become co-planar, where the $\mathrm{N}$ atom of the $\mathrm{NH}_{2}$ base has $s p^{2}$ hybridized orbitals, forming chemical bonds with two $\mathrm{H}$ atoms and a $\mathrm{N}$ atom at the top of the ring. The network of the $\pi$ orbitals in poly-aminotriazole is formed by those of the rings and that of $\mathrm{NH}_{2}$ 's. When the number of holes is decreased and becomes $n_{h}<1.1$, the $\mathrm{N}$ atom of the $\mathrm{NH}_{2}$ forms $s p^{3}$ hybridized orbitals, i.e., the $\pi$ orbital at $\mathrm{NH}_{2}$ disappears. Since the chemical bonds to the $\mathrm{N}$ atom and two $\mathrm{H}$ atoms are formed in the three directions of $s p^{3}$, leaving one lone pair, $\mathrm{NH}_{2}$ has a threedimensional structure. This time, the network consists of five $\pi$-orbitals of the rings and a $s p^{3}$ hybridized orbital of the lone pair. This change is disadvantageous for the scheme of the flat-band formation (Fig. 9) due to the balance of the six $\pi$ orbitals. This is considered why $\mathrm{F}$ state always takes a flat geometry while $\mathrm{P}$ state a non-flat geometry.

To see what part of the energy affects the change in the stable state when $n_{h}$ is decreased, we have calculated the energy difference between the $\mathrm{F}$ and $\mathrm{P}$ states by fixing the atomic structure to the optimum one for the $\mathrm{F}$ state at $n_{h}=2.0$. The result is plotted as a dotted line in Fig. 131 (a). This shows that the value is negative over the whole range plotted here, which indicates that the electronic part of the total energy always favors $\mathrm{F}$ state regardless of the filling of the flat band. Therefore, the change in the stable state at $n_{h}=1.1$ can be ascribed to the change in the structure, especially of $\mathrm{NH}_{2}$. If we can find a material in which there is no such change in the atomic structure but the parameter values are similar, we can expect an occurrence of the ferromagnetism even for a low-doping region less than quarter-filling.

In summary, the present work describes an example of materials design from first principles for the purpose of realizing flat-band ferromagnetism in organic polymers. We have found a promising material, polyaminotriazole, for which why it is the best and the robustness of the ferromagnetism has been discussed.

\section{Acknowledgments}

We would like to thank T. Hashizume, B. Choi, M. Ichimura and J. Yamauchi for fruitful discussions. We are also indebted to H. Nishihara and Y. Yamanoi for discussions on the chemistry of polymers. This study was performed through Special Coordination Funds for Promoting Science and Technology of the Ministry of Education, Culture, Sports, Science and Technology of the Japanese Government. The first-principles calculations were performed with TAPP (the Tokyo Ab-initio Program Package).
1 D. M. Eigler and E. K. Schweizer, Nature 344, 524 (1990).

2 T. C. Shen, C. Wang, G. C. Abeln, J. R. Tucker, J. W. Lyding, P. Avouris, and R. E. Walkup, Science 268, 1590 (1995).

3 T. Hashizume, S. Heike, M. I. Lutwyche, S. Watanabe, K. Nakajima, T. Nishi, and Y. Wada, Jpn. J. Appl. Phys. 35, L1085 (1996).

4 T. Hitosugi, S. Heike, T. Onogi, T. Hashizume, S. Watanabe, Z.-Q. Li, K. Ohno, Y. Kawazoe, T. Hasegawa, and K. Kitazawa, Phys. Rev. Lett. 82, 4034 (1999).

5 T. Ito, H. Shirakwa, and S. Ikeda, J. Polym. Sci. Polym.
Chem. Ed. 12, 11 (1974).

${ }^{6}$ H. Shirakawa, E. J. Louis, A. G. MacDiarmid, C. K. Chiang, and A. J. Heeger, JCS Chem. Commun., 578 (1977).

7 F. Garnier, R. Hajlaoui, A. Yassar, and P. Srivastava, Science 265, 1684 (1994).

8 R. H. Friend, R. W. Gymer, A. B. Holmes, J. H. Burroughes, R. N. Marks, C. Tailiani, D. D. C. Bradley, D. A. Dos Santos, J. L. Brédas, M. Lögdlund, and W. R. Salaneck, Nature 397, 121 (1999).

9 P.-M. Allemand, K. C. Khemani, A. Koch, F. Wudl, K. Holczer, S. Donovan, G. Gruner, and J. D. Thompso, Sci- 
ence 253, 301 (1991).

10 A. Rajca, J. Wongsriratanakul, and S. Rajca, Science 294, 1503 (2001).

11 A. Mielke and H. Tasaki, Commun. Math. Phys. 158, 341 (1993).

12 H. Tasaki, Prog. Thoer. Phys. 99, 489 (1998).

13 E. H. Lieb, Phys. Rev. Lett. 62, 1201 (1989).

14 N. Shima and H. Aoki, Phys. Rev. Lett. 71, 4389 (1993).

15 A. Mielke, J. Phys. A 24, 3311 (1991).

16 A. Mielke, J. Phys. A 25, 4335 (1992).

17 A. Mielke, Phys. Lett. A 174, 443 (1993).

18 H. Tasaki, Phys. Rev. Lett. 69, 1608 (1992).

19 H. Tasaki, J. Stat. Phys. 84, 535 (1996).

${ }^{20}$ H. Sakamoto and K. Kubo, J. Phys. Soc. Jpn. 65, 3732 (1996).

${ }^{21}$ R. Arita, K. Kuroki, H. Aoki, A. Yajima, M. Tsukada, S. Watanabe, M. Ichimura, T. Onogi, and T. Hashizume,
Phys. Rev. B 57, R6854 (1998).

${ }^{22}$ R. Arita and H. Aoki, Phys. Rev. B 61, 12261 (2000).

${ }^{23}$ K. Penc, H. Shiba, F. Mila, and T. Tsukagoshi, Phys. Rev. B 54, 4056 (1996).

24 J. P. Perdew, K. Burke, and Y. Wang, Phys. Rev. B 54, 16533 (1996).

25 D. Vanderbilt, Phys. Rev. B 41, 7892 (1990).

26 K. Laasonen et al., Phys. Rev. B 47, 10142 (1993).

27 R. Arita, Y. Suwa, K. Kuroki, and H. Aoki, Phys. Rev. Lett. 88, 127202 (2002).

28 J.-O Lee, G. Lientschnig, F. Wiertz, M. Struijk, R. A. J. Janssen, R. Egberink, D. N. Reinhoudt, P. Hadley, and C. Dekker, Nano Lett. 3, 113 (2003).

29 Y. Muraoka and Z. Hiroi, J. Phys. Soc. Jpn. 72, 781 (2003).

${ }^{30}$ R. Arita, Y. Suwa, K. Kuroki, and H. Aoki, submitted to Phys. Rev. Lett. 